\title{
Società, teatro e Servizio Sociale: la belleza è quello che ci salverà
}

\section{Society, theatre and Social Work: beauty will save us}

\author{
Simone SCHINOCCA \\ Director del Teatro Tedacá \\ direzioneteatro@tedaca.it
}

Recibido: 01/06/2015

Revisado: 07/07/2015

Aceptado: 15/12/2015

Disponible on line: 20/01/2016

\section{Riasunto}

Tre mondi differenti, a volte concentrici, a volte che si intersecano: la società, il teatro e l'arte performativa, e Servizio Sociale. Mondi diversi che vivono, specchiano, riflettono, interagiscono e possono avere occasioni di incontro/scontro e confronto e soprattutto offrire possibilità di profondo cambiamento. Un'esperienza di una compagnia teatrale che da tredici anni si muove ai confini di questi tre universi. E a questi tre mondi si aggiungono infinite parole che si riempiono di senso e significato: territorio, incontro, cultura, diversità, ricerca.

Una esperienza artistica che sceglie di concentrarsi e creare occasioni di dibattito e approfondimento sulle difficoltà, sulle umane contraddizioni, nel confronto costante e instancabile con l'esperienza umana. Tutto questo diventa cuore dell'agire teatrale ricercando un equilibrio fra racconto, incontro, ricerca e la dimensione artistica. E nell'incontro fra società, teatro e Servizio Sociale, ricercare anche una sostenibilità economica di una impresa culturale in un'Italia deflagrata dalla crisi, non solo economica, ma soprattutto di valori e punti di riferimento. Un filo conduttore, non sempre consapevole, ma sempre presente ed essenziale: la ricerca della bellezza.

Parole chiave: esperienza, bellezza, società, teatro e assistenza sociale.

\begin{abstract}
Three different worlds, sometimes concentric and often intersecting — society, theatre and the art of performance- and social work. Diverse worlds that live, reflect and self-reflect and interact, and can also afford an opportunity for meeting, misunderstanding and confrontation, and above all offer the possibility of profound change.

This article considers the experience of a theatre company that has spent more than three years moving at the limits of these three universes. To these three worlds can be added an infinite number of words that fill them with meaning and significance: territory, meeting, diversity and search. An artistic experience that has chosen to focus on creating scenarios for debate and to examine the difficulties, the human contradictions and the constant and inexhaustible confrontation with human experience. At the heart of this theatrical activity is all of this, seeking the balance between narration, meeting, investigation and the artistic dimension. This meeting between society, theatre and social work also contains the search for sustainability of this cultural business, in an Italy that has been destroyed by a crisis that is not merely economic, but also of values and, above all, of role models. The guiding theme, though not always made explicit, is always present and essential: the search for beauty.
\end{abstract}

Keywords: experience, beauty, society, theatre, social work.

\section{Resumen ampliado}

Tres mundos diferentes: la sociedad, el teatro y el Trabajo Social; a veces concéntricos y a menudo en intersección: la sociedad, el teatro y el arte de la performance y el Trabajo Social. Mundos diversos que viven, reflejan, se reflejan e interactúan, y que también son una ocasión de encuentro, desencuentro y confrontación, y sobre todo ofrecen la posibilidad de un cambio profundo.

En el artículo se relata y se reflexiona sobre la experiencia de una compañía de teatro, Tedacà, que lleva trece años moviéndose en los límites de estos tres universos; de estos tres mundos que encierran, además, una infinidad de palabras con un enorme significado: territorio, encuentro, cultura, diversidad y búsqueda. Se trata de una experiencia artística que ha optado por crear escenarios de debate y que profundiza en las dificultades, en las contradicciones humanas y en la confrontación constante e inagotable con la experiencia humana. En el corazón de esta acción teatral se busca el equilibrio entre narración, encuentro, indagación y la dimensión artística. En este encuentro entre sociedad, teatro y Trabajo Social se busca también la sustentabilidad de esta empresa cultural, en una Italia destrozada por la crisis, no solo económica, sino de valores y sobre todo de puntos de referencia.

La profesión del autor, como trabajador social, le ofrece una apertura de perspectivas: psicológica, sociológica y jurídica y un espacio de encuentro y afrontamiento con el mundo del Trabajo Social. Lucha para conciliar los tiempos de estudio 
con los laborales y el del itinerario teatral al que no puede reenunciar. Es una lucha en el terreno de lo que hasta entonces había sido el mundo de la enfermedad mental, o el mundo de la cárcel, cuando como consultor en la cárcel de Ivrea, participó en un proyecto de acompañamiento a personas que van a salir de la cárcel, una vez cumplida la pena, y que tienen un pasado de drogadicciones. Es la forma de estar en el mundo del trabajador social, es como llevar una alianza en el dedo. El arte, la sociedad y el Trabajo Social se han encontrado en muchos proyectos: con todo tipo de escuelas, con el problema del acoso y de la diversidad; proyectos que tratan de la exclusión y que relatan la profunda violencia, el sufrimiento y la soledad que se encierran en estas dimensiones. Ha tratado también el tema de acogimiento familiar, trabajando con los niños para llegar a sus padres. Ha realizado talleres de arte para toda la ciudadanía, con los jóvenes, usuarios de los Servicios Sociales de los pisos tutelados, sin crearles itinerarios ad hoc, sino tratando de insertarlos en los de sus coetáneos, tratando de eliminar así cualquier etiqueta que pudiera diferenciarlos, y simplemente buscando el encuentro entre ellos. El hilo conductor no siempre era consciente, aunque siempre ha estado presente, porque era tan esencial como lo es la búsqueda de la belleza.

Palabras clave: experiencia, belleza, sociedad, teatro, Trabajo Social.

Referencia normalizada: Schinocca, S. (2016): «Società, teatro e Servizio Sociale: la belleza è quello che ci salverà». Cuadernos de Trabajo Social, 26(1): 43-50.

Sumario: 1. Presentazione. 2. Metodo. 3. Risultati. 4. Discussione. 5. Riferimenti bibliografici.

\section{Presentazione}

Tutto inizia spesso in una strada di inconsapevolezza.

Come fare a sapere che tutto andrà come deve andare e sapere, con il senno di poi, che quello che stai vivendo non è semplice esperienza, ma un mattone delle fondamenta del tuo futuro pensare, agire e vivere?

A volte la sentiamo la consapevolezza del collocare un mattone importante per quello che sarà. Alcuni mattoni, poi, non si rivelano tali, altri inaspettati, diventano chiave di volta del futuro.

E' la nostra storia che ci dà la capacità di discernere.

Un ragazzo quasi alla soglia dei vent'anni. Una famiglia alle spalle semplice, semplicemente buona che è arrivata a Torino, da qualche decennio, dalle campagne di un Sud Italia spesso dimenticato.

Nello zaino un percorso di studi più scientifico che umanistico, anni nel mondo del volontariato e dell'associazionismo. E proprio da quei mondi nasce il desiderio di studi universitari che abbandonino la chimica, la fisica e la matematica e che diano strumenti di lettura dell'oggi, della società e soprattutto delle sue contraddizioni e difficoltà.

Una laurea in Servizio sociale offre aperture ed esplorazioni nel mondo della psicologia, della sociologia, del diritto e di incontro e confronto con il mondo del lavoro sociale, permettendo di assumere un habitus interdisciplinare e interprofessionale che si rivela fondamentale nell'approcciare feno- meni e nel gestire relazioni e che è connaturato alla cultura del servizio sociale (Gui, 2004).

Una passione che esplode non tanto nelle procedure e nella dimensione di aiuto, ma soprattutto nell'infinita possibilità che il Lavoro Sociale offre di conoscere le contraddizioni del nostro tempo, i confini, le storie di limiti e fatiche inimmaginabili. E proprio in quelle zone di confine, in quelle terre spesso di nessuno, così lontane da proiettori e attenzioni, trovare una dimensione di senso, di ricerca, di possibilità di lettura e interpretazione del tempo presente (Manicardi, 2012).

A tutto questo si affianca una passione indomabile, incontrollata, per il mondo del teatro. Un percorso di formazione lungo e faticoso nell'arte performativa cha affianca e a volte combatte con i tempi universitari e con le prime esperienze lavorative nel mondo del sociale.

Con un percorso di studi giunto poco oltre la metà, subentra per l'appunto il mondo del lavoro. Prima occupazione una comunità che lavora con giovani affetti da malattie psichiatriche. Prima terra di confine. La lotta fra il conciliare i tempi del mondo accademico, i turni del lavoro, e il percorso teatrale inabbandonabile. E la lotta nel confronto fra quello che è stato fino a quel momento e il mondo delle malattie psichiatriche. Termina il percorso di studi, avviene l'iscrizione all'Albo degli Assistenti Sociali. L'Albo non è un semplice registro. E' un modo per affermare al mondo che lo si è Assistenti Sociali. E' una fede al dito. Io l'ho vista sempre così. 
Si affianca al mondo della psichiatria una consulenza al carcere di Ivrea su di un progetto di accompagnamento alle persone in prossimità di fine pena, con esperienze nel passato di tossicodipendenze. Un altro confine, un'altra terra di nessuno, a volte. Il carcere. Le sue regole. La bellezza, l'incontro e lo scontro del lavorare con altre professioni: educatori, medici, psicologi.

E il teatro sempre li, ad affiancare tutto questo. Ma vissuto fino a quel momento come un mondo separato, che non andava «contaminato» con le esperienze lavorative. Forse in quella fase, così intensa di formazione e collocazione di mattoni fondamentali per il futuro, era inevitabile tenere tutto profondamente separato.

E poi il concorso pubblico, e l'arrivo dell'incarico in un Centro di Servizi Sociali di territorio, periferia estrema, palazzoni stracolmi di famiglie «multiproblematiche». Che termine assurdo, che indica semplicemente la scelta per decenni di confinare in un unico luogo, famiglie e situazioni al confine, al limite, stracolme di profonde difficoltà. Un susseguirsi di palazzi di dodici piani, brutti, senza alcuna logica, confinati, e in qualche modo anche poco visibili dalla città. E' li, il servizio sociale in cui arrivo. E infinite storie, infinita umanità, in cui la bellezza semplicemente è celata ma profondamente presente.

Un assistente sociale anomalo. E lì esplode tutto. Il pensiero, il desiderio che quell'infinita umanità, che l'umano con le sue fatiche e contraddizioni, debba trovare uno spazio di racconto. Sapevo fare Teatro. Ne avevo il mezzo. Ne avevo l'urgenza

Nasce Tedacà, una compagnia teatrale di cui sono fondatore. Tedacà in aramaico significa giustizia. Ma quando l'abbiamo creata non lo sapevamo. I mattoni inconsapevoli. Per noi era solo l'acronimo di Teatro, Danza, Canto, con l'accento, perché così suona meglio. Fin dalla fondazione si svela quello che sarà il cuore. Lo Statuto afferma

Tedacà ha come scopo principale l'espressione dell'umano attraverso l'uso delle arti della scena e delle arti in generale. Nell'Associazione, il Teatro, la Danza il Canto, e le forme espressive in genere sono strumento fondamentale di racconto e testimonianza dell'uomo, delle sue difficoltà e contraddizioni e sono possibilità di confronto, scam- bio e crescita con la realtà, nelle sue innumerevoli espressioni, che circonda ciascuna persona». ${ }^{1}$

Un luogo fisico e mentale di incontro fra arte e società. L'arte con la sua profonda necessità di connettersi, astrarsi e restituirsi alla Società. La Società con la necessità di luoghi e dimensioni in cui specchiarsi, con la necessità di individuare metafore e racconti in cui potersi guardare da fuori (Stanislavkij, 1963). La ricerca di altri punti di vista e prospettive. La possibilità di spazi onirici in cui aspirare al meglio, riscoprire capacità di desiderio e prospettive possibili. Luoghi e dimensioni in cui scegliere di cambiare o di non volere più.

E in tutto questo il lavoro sociale da cui provenivo che entra in contatto con gli angoli più bui, spesso più faticosi, più dimenticati, più contradditori. Con le sue continue urgenze.

Urgenza parola fondamentale anche per il mondo teatrale e dell'arte, con un significato solo apparentemente diverso, rispetto al mondo del lavoro sociale. L'urgenza è il motivo, motore, punto di partenza per cui una creazione artistica prende il via (Bruschetta, 2010). Senza urgenza non potrebbe esserci arte. E fra Arte, Società e Lavoro Sociale cercare un incontro. Da tredici anni. Un incontro fra le urgenze, da diverse prospettive

\section{Metodo}

Tutto è inconsapevole a volte. E inconsapevole è stato l'incontro con altri artisti provenienti dal mondo della danza e della musica che avevano nel loro percorso, formazione, attenzione, cura ed esperienze nel lavoro sociale. E' appunto la nostra storia che ci dona capacità di discernere $\mathrm{e}$ disponibilità all'incontro. Dall'incontro con questi artisti l'idea diventa possibilità. Tedacà diventa realtà.

Da quel momento divento Regista sulla carta di identità. Assistente sociale lo rimango nel modo di pensare e guardare a ciò che mi circonda. Il mio strumento: il teatro.

E' tutto gioco, leggerezza, sfrontataggine, assolutamente non rispetto dei canoni e della serietà che l'arte spesso impone come suo bigliettino da visita, che spesso diventa più forma che sostanza. La giovane età, mia e di tutti coloro che si buttarono in questa avventura, la faceva da pa-

\footnotetext{
${ }^{1}$ Statuto Associazione Tedacà, Torino, 2002.
} 
drone. venticinque anni sono proprio un'età leggera.

Pian piano alcune parole diventano guida e cuore del lavoro della compagnia.

Territorio. Una compagnia, un gruppo di persone e artisti che lavorano in mezzo alla gente. Con scuole, Servizi Sociali, cooperative e associazioni, che creano spazi di informalità e incontro con i vicini, con le famiglie residenti, con i negozianti. Il territorio come luogo di incontro, ascolto e possibilità. E il territorio è da sempre nel servizio sociale un elemento cruciale, dove si ricompongono bisogni e risorse e dove, all'assistente sociale, è data la possibilità di promuovere risorse per le persone ma anche con le persone, favorendo il loro protagonismo e non considerandole solo «utenti» in condizione di bisogno (Guerrini, 2013)

Casa. Una casa per la nostra compagnia l'abbiamo cercata fin da subito. Prima uno scantinato affittato da una cooperativa sociale. Lo abbiamo sistemato, dipinto e fatto nostro. Ai nostri occhi era bellissimo. Poi, qualche anno dopo, uno spazio messo a disposizione dalla città. Era una fabbrica, noi l'abbiamo trasformato in un teatro, in uno spazio polivalente, punto di riferimento di un territorio. Uno spazio, aperto un'infinità di ore, capace di ospitare una stagione teatrale, prove di compagnie e gruppi, progetti, mostre, laboratori, attività, pranzi e feste.

Cultura diffusa. In queste due parole sta il cuore. La cultura per noi è sempre stata una parola che andava portata in mezzo alla gente, per la gente. Non frequentiamo salotti culturali. Cerchiamo di portare occasione di cultura su terreni e territori spesso dimenticati. La cultura deve essere capace di parlare con la gente, di ascoltare le istanze della gente. La cultura non deve avere paura di andare verso i luoghi dei cittadini, case, periferie, scuole, discoteche, centri commerciali... Deve andare verso. E più si orienta verso chi ha meno occasioni e più può diventare interessante, importante, utile, vera.

Apertura. La nostra fabbrica, trasformata in teatro ha tantissime finestre che danno sulla strada. Chi cammina può vedere tutto quello che capita dentro. Spesso le scelte sono inconsapevoli ma figlie della nostra storia. In questi anni abbiamo sempre lottato per tenere costantemente aperta una porta su quello che ci circonda. Cittadini, istituzioni, eventi, crisi, attenzione all'oggi, alle sue urgenze e necessità. Infiniti i progetti ac- colti, le realtà con cui si sono create collaborazioni, le istanze che abbiamo ascoltato e di cui abbiam provato ad esser portavoce.

Contraddizioni e fatiche. Da li parte la nostra urgenza. Dal raccogliere, vedere, raccontare, trasformare, dibattere e sognare su quello che affatica le nostre vite, che ci coglie impreparati, che ci spiazza, che ci addolora. Usare i linguaggi dell'arte come strumento di racconto di tutto questo. Come possibilità di astrazione, riflessione. Come occasione del porre delle domande, che auspichino e possano innescare un cambiamento, una differente prospettiva.

Cura. Una parola così dimenticata dal nostro tempo. Il lavorare con cura. L'avere cura. Il curare i propri affetti. Aver cura dell'umano che ci circonda e che incontriamo. Una parola fondamentale, una attitudine e attenzione che con il tempo è diventata consapevolezza, elemento che ci contraddistingue, che fa parte di noi e del nostro tentativo di agire. L'idea della cura è fortemente presente nel servizio sociale che, all'interno del processo di aiuto (Cellini e Dellavalle, 2015), si prende cura della persona, senza dimenticarsi di prendersi cura di sé, attraverso la formazione continua (CNOAS, 2014), la riflessività e la disponibilità ad apprendere dagli errori (Sicora, 2010), in un circolo virtuoso dove curarsi del proprio essere professionista qualifica l'intervento e garantisce la cura autentica della persona.

E da tutte queste parole in tredici anni, Arte, Società e Lavoro Sociale si sono incontrati in infiniti progetti.

Lavorando moltissimo con scuole di ogni ordine e grado è stato naturale immaginare, concentrarsi e realizzare molti progetti sui fenomeni del bullismo, dell'accettazione e rispetto della diversità, progetti che cercano di contrastare fenomeni di emarginazione, che raccontano la profonda violenza, sofferenza e solitudine che spesso si cela in queste dimensioni.

Abbiamo parlato, con uno spettacolo intitolato Shamandura, del delicato tema dell'affidamento famigliare, lavorando con i bambini delle scuole elementari per poter arrivare ai loro genitori, per insinuare il dubbio e il desiderio di aprire le porte della propria casa a bambini provenienti da famiglie in difficoltà. Shamandura è una parola che usano i pescatori. E' quella zona nelle tempeste, dove l'infrangersi delle onde contro gli scogli crea una striscia di mare in cui, 
pur rimanendo nella burrasca, è possibile prendere fiato, per poter poi ripartire.

Abbiamo aperto i laboratori artistici rivolti ai cittadini, a giovani seguiti dai Servizi Sociali o inseriti in comunità alloggio per Adolescenti. Non andando a creare dei percorsi ad hoc, ma inserendo i ragazzi nei percorsi frequentati dai loro coetanei, cercando di eliminare qualunque etichetta e favorendo semplicemente l'incontro.

Abbiamo aperto le porte della stagione teatrale, da noi organizzata, ad associazioni che lavorano con anziani e con persone diversamente abili. Cercando di creare occasione di fruizione e partecipazione agli spettacoli, favorendone l'accesso, convogliando giovani volontari nell'accompagnamento e nella cura all'accoglien$\mathrm{za}$, organizzando spettacoli anche in orari inconsueti e più accessibili a persone, che dopo cena, incontrano difficoltà nell'uscire da casa. E la stagione è caratterizzata da spettacoli e performance che raccontano con prospettive e linguaggi diversi le difficoltà del nostro tempo presente. $\mathrm{La}$ stagione si chiama da due anni Nova Vita. Occasioni di rinascita, ripensamento, cambiamento, possibilità di prospettive differenti.

Infinite le tematiche su cui abbiamo organizzato, ospitato progetti. Omoaffettività, emigrazione, lavoro, emancipazione dell'universo femminile. Da sette anni organizziamo una stagione estiva, in un parco bellissimo della città, i Giardini Reali. Venti giorni in un clima informale, dove mangiare cibo, preparato con cura, e assistere a performance e spettacoli a ingresso gratuito. Filo conduttore della rassegna il tema della salute e del benessere. In una accezione allargata. Ogni giorno un tema diverso e prima dello spettacolo l'incontro e il racconto con realtà, servizi, istituzioni, associazioni che lavorano su quel tema. Nell'ultima edizione oltre settanta realtà raccontate a centinaia di persone che si radunano sotto il palco, complice la piacevolezza del luogo e il desiderio di occasioni di socialità nel caldo estivo di una grande città.

$\mathrm{E}$ da tutto questo lavorare in una dimensione di incontro fra Arte Società e Lavoro Sociale, gradualmente è diventato consapevole un ulteriore passaggio, ormai caratteristica della nostra compagnia teatrale. L'utilizzare questi percorsi di incontri, come cuore della ricerca della compagnia teatrale, delle sue produzioni e delle creazioni degli spettacoli destinati alla circuitazione nazionale.

Attori e performer professionisti che dall'incontro con il mondo del sociale portano in scena, in produzioni destinate alla circuitazione nei diversi teatri, storie, racconti, temi e difficoltà del nostro tempo. Produzioni che sono state multi premiate e che hanno ottenuto consenso di pubblico e critica

Da quaranta storie/interviste di persone emigrate dal Sud al Nord Italia fra gli anni Cinquanta e Settanta nasce un copione Strani-ieri, che molto fa riflettere sull'emigrazione anche dei nostri giorni. Da un blog dove chiunque poteva raccontare storie di disparità fra uomini e donne sul mondo del lavoro, nasce Pari \& Dis-pari, spettacolo ed evento cuore di Biennale Democrazia $^{2}$. Da un percorso di formazione sulla narrazione, per educatori professionali che lavorano con adolescenti in una comunità alloggio, nasce Street Striit favola coreutica, che racconta di quanto spesso possiamo non sentirci pronti nella nostra vita, ad esempio, nell'affrontare una maternità. Una bambina che nasce da una mamma ne buona ne cattiva, semplicemente non pronta, cresce e più cresce e più la sua rabbia aumenta, tanto che ad un certo punto, si trasforma in un burattino a cui si ingarbugliano i fili e non riesce più a districarli. Questa favola metafora scritta dagli educatori diventa non solo uno spettacolo teatrale ma anche un fumetto, distribuito a tutto il pubblico, che racconta come proprio attraverso il lavoro sociale pian piano quei nodi possano sciogliersi.

Proprio in questi mesi stiamo lavorando ad una performance di massa che ha l'intento di denunciare le nuove forme di povertà dilaganti nella nostra società, avendo come punto cardine anche per la messa in scena, il concetto della dignità.

Il lavorare su testi classici e della tradizione del teatro provando a trovare innesti e confronti con l'attualità e le domande del nostro tempo. E allora portare in scena un Otello, con Jago e il suo profondo desiderio di autorealizzazione capace di distruggere tutto e qualsiasi cosa, diven-

2 Manifestazione di interesse nazionale organizzata biennalmente a Torino e in cui importanti ospiti nazionali e internazionali del mondo della cultura, della politica, del diritto, del sociale sono chiamati a confrontarsi su importanti temi legati alla Democrazia. 
ta specchio dell'individualismo in cui siamo sommersi. E allora la messa in scena si riempie e racconta il nostro tempo, le nostre fatiche, $i$ nostri limiti.

Infinite strade e lavori. Infiniti incontri con cooperative sociali, Servizi Sociali, Università, centri di formazione professionale, operatori sociali, volontari. Tutto questo è infinitamente lontano rispetto al luogo comune del pensiero di un artista chiuso in uno spazio, buio e poco raggiungibile, dove crea. Il nostro teatro, pieno di finestre, e la nostra arte parte, vive e si confronta in mezzo alla gente.

\section{Risultati}

Tredici anni di vita.

Tredici anni di Esperienza teatrale come specchio della vita. Indubbiamente in tutti questi anni un risultato importante è il riavvicinamento della gente, del pubblico alla dimensione teatrale. Il nostro teatro è frequentato da oltre diciottomila persone all'anno. Un risultato incredibile per uno spazio di periferia. Il pubblico si riavvicina perché riesce a vivere con empatia quello che succede sul palco. Perché vi vede riflessa la propria vita, ne trova domande, spunti di riflessione, luoghi e spazi di pensiero.

Forse il risultato più importante è l'esser riusciti a dar voce a tematiche e fatiche della nostra contemporaneità, creando occasioni di conoscenza e dibattito pubblico. L'aver portato tematiche importanti e urgenti del lavoro sociale, al di fuori delle «mura» dei servizi. Averle portate in «piazza», in teatro, al pubblico che spesso non conosce e non ne ha consapevolezza. Far scoprire parti di mondo e in particolare le difficoltà che spesso vivono pezzi della nostra società, ad un pubblico allargato, a giovani, al mondo delle scuole creando semi di dibattito e riflessione futura.

Parlare di emigrazione, immigrazione, affidamento famigliare, del prendersi cura, delle difficoltà dell'universo giovanile, di solitudine, del senso di inadeguatezza diffuso, del desiderio di cambiamento è stato il nostro modo di fare cultura in questi anni e di parlare confrontarci con il pubblico. Con il passare degli anni, l'audience è considerevolmente aumentato, dimostrando anche quanto le persone siano desiderose nel incontro con il mondo della cultura, di sentire parlare, dibattere e confrontarsi con le difficoltà e le possibilità della nostra contemporaneità.
Lavorare sulla rilettura del tempo presente, avendo cura e attenzione a far emergere la dimensione di desiderio, spesso assopita e nascosta nella nostra società. E non solo desiderio, ma la ricerca di Bellezza, come cuore e chiave di lettura del nostro tempo e delle nostre vite. Anche nel confine più nascosto, nell'immensità di un buio soffocante, possiamo provare a individuare spazi di bellezza che possono essere il punto da cui ripartire, affrontare, interrogarsi e provare ad agire azioni di cambiamento. La Bellezza come luogo da rilevare, da scovare, individuare e da cui partire.

Bellezza è l'incontro, sono le capacità residue, le capacità di resilienza, i punti di forza, le possibilità di rete e di aiuto, gli spazi di collaborazione, le aperture, le storie, le esperienze. Ogni storia, ogni incontro può avere infinite possibilità di bellezza.

Arte e la cultura devono aiutare la società e chi lavora per migliorare il nostro tempo, come fanno quotidianamente i lavoratori del «sociale», a individuare possibili spazi di bellezza. Sostenere l'incontro e il confronto, dare apertura, offrire apertura. Essere Agorà. Una piazza dove si incontrano vite, storie, spesso nascoste, sfaccettature inimmaginate, $\mathrm{e}$ in queste provare a individuare riflessioni e domande che possano essere capaci di cambiare il nostro tempo presente per donare posti e tempi migliori alle generazioni future. Retorica? Possibilità. Per il nostro lavoro senso delle quotidiane fatiche. Per noi Arte e cultura non avrebbero senso se non in questa accezione.

\section{Discussione}

Molte le domande aperte cui ogni giorno proviamo a individuare possibili risposte.

Tutto questo si scontra e fa i conti quotidianamente con la dimensione dello «snobbismo culturale». Questa modalità operativa, quanto costruito, non ha nulla a che vedere con i salotti di cui vive e si alimenta la «cultura» dominante. La diffidenza dei templi della cultura è spesso tangibile. Solo il tempo, la serietà, i risultati, il pubblico, riescono ad aprire un dialogo e spazi di rappresentazione e valorizzazione con i circuiti ufficiali.

Bisogna essere capaci di resistere. E su questa dimensione il lavoro sociale molto $\mathrm{mi}$ ha insegnato.

Tutto questo deve trovare una dimensione di sostenibilità economica. Ormai la nostra compagnia è una vera e propria impresa culturale: nove addetti in pianta stabile, quindici collaboratori, oltre 
trecento soci e due sedi. La dimensione della sostenibilità economica è elemento fondamentale soprattutto in un periodo in cui i finanziamenti pubblici sono sempre più simbolici, sporadici, erogati con un ritardo inimmaginabile. La capacità di differenziazione nella progettazione, la capacità di relazione con molti soggetti, sono ad oggi una possibilità di individuazione di strade per la sostenibilità economica. La nostra realtà non avrebbe potuto reggere e così svilupparsi senza queste capacità e caratteristiche acquisite nel tempo.

Infiniti i temi che potremmo affrontare e su cui la nostra società quotidianamente ci sollecita. Alcuni temi del nostro prossimo futuro: la dimensione della precarietà lavorativa, sociale, affettiva. La dimensione del potere e dello scontro generazionale. L'emigrazione e la ricerca di «Terre promesse». A volte si ha la sensazione di lavorare con i vasi di Pandora. Apri un tappo e sfociano infiniti mondi. E su questo quotidiana fatica per trovare una giusta ed equilibrata dimensione di racconto. Non smarrirsi, non perdersi, non farsi fagocitare.

La costante ricerca di aperture, e non di soffocamento. Di buone pratiche, di originalità, di risposte positive nelle esperienze e non fermarsi alla semplice denuncia dei problemi. L'affrontare le diverse tematiche cercando di individuare possibili prospettive. Non è facile, richiede immane fatica. Ma è proprio in quella fatica che ha senso il lavoro artistico.

La ricerca di ulteriori confini, di nuove terre d'ombra, di assenze di diritti. Esperienze inconsuete, nascoste che interrogano ancora di più, il nostro tempo e la nostra società. Quello che intorno a noi esiste, ci parla. Ma la ricerca si deve spostare forse ancora di più, su terreni poco esplorati, su fatiche immani che spesso sono nas- coste, non raccontate, di cui spesso abbiamo ancora più timore. In questi mesi molti di noi sentono questa urgenza, di premere ulteriormente il piede sull'acceleratore nello spingersi alla ricerca dei confini estremi e delle zone d'ombra della nostra società. E questo atteggiamento, questa spinta alla ricerca di nuovi spazi e nuovi confini, che ha caratterizzato anche la stessa professione del servizio sociale italiano negli anni del secondo Dopoguerra e della Ricostruzione (Stefani, 2012; Appetecchia, 2015), dovrebbe ritornare ad animarla oggi, in un contesto dove la crisi globale e il welfare mix hanno modificato $i$ rapporti fra servizi pubblici, mercato, Terzo settore, e l'emergere di nuove forme di povertà e vulnerabilità richiede innovazione anche sul piano professionale. L'impatto delle trasformazioni rischia di ridurre la figura dell'assistente sociale ad una pedina burocratica e questo può essere contrastato con l'assunzione di funzioni di promozione, informazione, sensibilizzazione all'internodi un sistema di risposte che rispetti la dignità umana e i diritti (Dellavalle, 2013).

$\mathrm{E}$ in tutto questo nostro lavoro, in questa ricerca di nuovi limiti e confini, non possiamo non considerare, l'inalienabile dimensione di solitudine in cui i lavoratori sociali e allo stesso modo gli artisti si immergono. Spesso ci si sente soli. $\mathrm{Si}$ anela ad un confronto con qualcuno che possa comprendere, che possa con noi vivere con empatia quella dimensione di confine. Perché il confine ti ingloba, rischia di triturarti ed escluderti allo stesso identico modo di chi lo vive. Un'attenzione sempre presente. Di nuovo Cura questa volta di sé e del proprio animo.

La bellezza. Come punto di partenza. Come occasione di riflessione e ripensamento. Come speranza e desiderio di futuro.

\section{Riferimenti bibliografici}

Appetecchia, E. (2015) (a cura di). Idee e movimenti comunitari. Servizio sociale di comunità in Italia nel secondo dopoguerra. Roma: Viella.

Bauman, Z. (2000). Il disagio della postmodernità. Milano: Bruno Mondadori.

Bruschetta, N. (2010). Sul mestiere dell'attore. Milano: Bompiani.

Campanini, A. (2013), (direzione di). Nuovo Dizionario di servizio sociale. Roma: Carocci.

Cellini, G. e Dellavalle, M. (2015). Il processo di aiuto del servizio sociale, Prospettive metodologiche. Torino: Giappichelli.

CNOAS. (2014). Regolamento per la formazione continua. Roma: Consiglio Nazionale Ordine Assistenti Sociali. Reperibile a questo link: http://www.cnoas.it/cgi-bin/cnoas/out.pdf?i=IIFIRINILGKITIGIXFAAGC\&e=.pdf\&t=normazione (u.a. 12 dicembre 2015).

Dellavalle, M. (2012). Persistenze: democrazia e diritti di cittadinanza nel percorso del servizio sociale. La Rivista di Servizio sociale, 2, 36-43. 
Grotowsky, J. (1970). Per un teatro povero. Roma: Bulzoni Editore.

Guccini, G. (2005). La bottega dei narratori. Roma: Dino Audino Editore.

Guerrini, M.R. (2013). Territorio. In A. Campanini (direzione di), Nuovo Dizionario di servizio sociale (pp. 714-717). Roma: Carocci.

Gui, L. (2004). Le sfide teoriche del servizio sociale. Roma: Carocci.

Manicardi, L. (2012). Raccontami una storia. Narrazione come luogo educativo, Padova: Edizioni Messaggero.

Moscati, I. (1999). Luca Ronconi: utopia senza paradiso. Sogni disarmati al laboratorio di Prato. Venezia: Marsilio.

Schino, M. (2009). Alchimisti della scena, teatri laboratorio del Novecento Europeo. Bari: Edizioni Laterza.

Sicora, A. (2010). Errore e apprendimento nelle professioni di aiuto: fare più errori per fare meno danni? Santarcangelo di Romagna: Maggioli.

Stanislavkij, K. (1963). Il lavoro dell'attore su se stesso. Roma: Edizioni Laterza.

Stefani, M. (a cura di). Le origini del Servizio sociale italiano. Roma: Viella. 